\title{
Transmitter Localization in Vessel-like Diffusive Channels using Ring-shaped Molecular Receivers
}

\author{
Meriç Turan, Bayram Cevdet Akdeniz, Mehmet Şükrü Kuran, H. Birkan Yilmaz, \\ Ilker Demirkol, Ali E. Pusane, and Tuna Tugcu
}

\begin{abstract}
Molecular communication via diffusion in vessellike environment targets critical applications such as detection of abnormal and unhealthy cells. In this work, we derive the analytical formulation of the channel model for diffusion dominated movement, considering ring-shaped (i.e., patch) observing receivers and Poiseuille flow with the aim of localization of the transmitter cell. Then, we derive formulations using this channel model for two different application scenarios. We assume that the emission start time is known in the first scenario, and unknown in the second one. We successfully localize the transmitter cell using a single receiver for the first scenario, whereas two receivers are used to localize the transmitter cell in the second scenario. Lastly, the devised analytical framework is validated with simulations.
\end{abstract}

Index Terms-Molecular communication via diffusion, vessellike environments, channel modeling, localization.

\section{INTRODUCTION}

Molecular communication via diffusion (MCvD) is one of the prominent communication systems in the greater context of nanonetworking, which aims to enable communication between cell-sized machines in in vivo applications. In $\mathrm{MCvD}$, information is carried over special messenger molecules (MMs) between the transmitter and the receiver cells, all of which reside inside a fluid environment [1]. Until recently, most of the works focusing on MCvD systems have assumed an unbounded free diffusion environment that consists of only the communicating pair and the MMs used for communication. Although such environments are extremely suitable for analytical analysis of the various aspects of this unique communication system, their applicability is somewhat limited for biomedical applications.

A more realistic alternative to the unbounded free diffusion environment is the vessel-like environment (VLE) [2], which is composed of a cylindrical environment resembling a blood vessel encompassing all the components of the system. Additionally, there is a flow element which also affects the movement of the MMs. These differences change the channel

This work was supported in part by TUBITAK under grant number 116E916 and TETAM under grant number DPT-2007K120610, by the Government of Catalonia's Secretariat for Universities and Research via the Beatriu de Pinós postdoctoral programme, and by ERDF and the Spanish Government through projects RYC-2013-13029 and TEC2016-79988-P. M. Turan is the corresponding author.

M. Turan and T. Tugcu are with Dept. of Computer Engineering, NETLAB, Bogazici University, Istanbul, Turkey (e-mail: meric.turan@boun.edu.tr). B. C. Akdeniz and A. E. Pusane are with Dept. of Electrical and Electronics Engineering, Bogazici University, Istanbul, Turkey. M. S. Kuran is with Dept. of Computer Engineering, Abdullah Gul University, Kayseri, Turkey. H. B. Yilmaz and I. Demirkol are with Dept. of Network Engineering, Universitat Politecnica de Catalunya, Barcelona, Spain. model of the MCvD system considerably. In the literature, several communication topologies are proposed for such environments based on the type of the receiver and the nature of the flow. Receivers can be absorbing, observing or temporarily binding with respect to the MMs passing through them, while being either fully or partially covering the cross section of the cylindrical environment. Moreover, the flow can be either non-existent [3], uniform [4], or laminar [5].

In the molecular communication literature, some former works have focused on distance estimation between the transmitter and receiver cells. In [6] and [7], distance estimation in a 1-D unbounded environment with no flow is achieved by utilizing the peak concentration. In [8], several channel parameters, including distance, have been estimated using a maximum likelihood estimation (MLE) method considering a 3-D unbounded environment with a uniform flow. In a similar work, Lin et al. have also proposed using MLE to estimate channel parameters in a 1-D diffusive channel with flow using the release time of molecules, which is assumed to be encoded inside the MMs [9]. To the best of our knowledge, distance estimation inside a VLE with Poiseuille flow has not been examined in the literature. In this work, we study the channel model of such a VLE in the MCVD system for diffusion dominated movement as well as develop an analytical model to localize the transmitter, where the transmitter's location is unknown to the receiver. We focus on the transmitter localization problem, since it is critical for several MCvD applications, such as detection of abnormal or unhealthy cells in the vicinity of the capillaries. We consider multiple ring-shaped receivers (i.e., patch receivers) with observing characteristics and a flow component that resembles the blood vessel environment. In the literature, various flow models are used for such environments considering different types of blood vessels. In this paper, we focus on Poiseuille flow, which defines the flow profile in blood vessels whose radii are not varying [10], [11].

As such, the main contributions of this letter are:

- We develop a channel model of the MCvD system in a VLE considering a point transmitter, ring-shaped observing receivers, and Poiseuille flow.

- We derive analytical formulations to estimate the transmitter location by a single ring-shaped receiver when the emission start time is known by the receiver.

- We also derive analytical formulations to estimate the transmitter location by two ring-shaped receivers when the emission start time is not known by the receivers. 


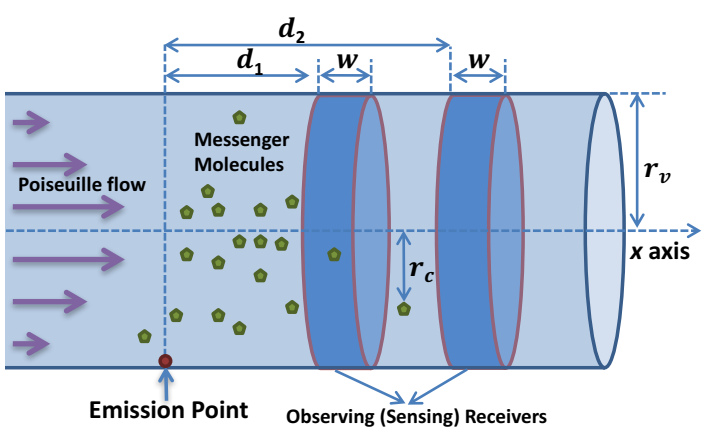

Fig. 1. Micro-fluidic based communication channel representation. Derived formulations are independent from the radial location of the emission point.

\section{SySTEM MODEL}

In this work, we focus on a VLE composed of a single point transmitter and multiple fully observing ring-shaped receivers, where a positive Poiseuille flow exists towards the receivers (Fig. 1). The Poiseuille flow is a type of parabolic flow that represents the pressure induced flow of an incompressible viscous fluid in a long narrow duct. The ring-shaped receivers are located on the vessel perimeter having a radius $\left(r_{v}\right)$ that is the same as the vessel's radius and a certain width $(w)$. The receivers sense either their whole projection area or up to a certain depth. The vessel is considered to be a perfect cylinder with a fully reflecting inner surface.

\section{A. Diffusion Model}

In the diffusion model, the total displacement along the flow direction $(\Delta X)$ of an MM during one simulation time step $(\Delta t)$ has two dominant factors: displacement due to the diffusion ( $\left.\Delta X_{\text {diffusion }}\right)$ and displacement due to the flow ( $\Delta X_{\text {flow }}$ ). Displacement due to the diffusion follows a Gaussian distribution with

$$
\Delta X_{\text {diffusion }} \sim \mathscr{N}(0,2 D \Delta t),
$$

where $D$ is the diffusion coefficient and $\mathscr{N}\left(\mu, \sigma^{2}\right)$ is the Gaussian distribution with mean $\mu$ and variance $\sigma^{2}$. Displacement due to the flow is calculated as

$$
\Delta X_{\text {flow }}=v_{f}\left(r_{c}\right) \Delta t
$$

where $v_{f}\left(r_{c}\right)$ is the Poiseuille flow velocity [12] and

$$
v_{f}\left(r_{c}\right)=2 v_{m}\left(1-\frac{r_{c}^{2}}{r_{v}^{2}}\right),
$$

where $v_{m}$ is the average flow velocity and $r_{c}$ is the distance between MM and the central axis of the cylinder.

Since the flow is not turbulent, it does not have any effect on the other two axes. Therefore, the movement in the $y$ and the $z$ axes are purely diffusion oriented. Consequently, the total displacement in all three axes in a single time step is

$$
\Delta \vec{r}=\left(\Delta X_{\text {diffusion }}+\Delta X_{\text {flow }}, \Delta Y_{\text {diffusion }}, \Delta Z_{\text {diffusion }}\right),
$$

where $\Delta Y_{\text {diffusion }}$ and $\Delta Z_{\text {diffusion }}$ correspond to the displacement in the $y$ and the $z$ axes, respectively, both of which follow a Gaussian distribution as in (1).
Another important factor while considering diffusion is the ratio between the advection and the diffusion. This ratio is defined by the Péclet number as

$$
\mathrm{Pe}=\frac{\text { advection transport }}{\text { diffusion transport }}=\frac{v_{m} r_{v}}{D} .
$$

As can be seen in (5), the movement is more diffusion based for smaller Pe values and vice versa (e.g., $\mathrm{Pe}=0$ represents pure diffusion). In this paper, we consider diffusion dominated movement, so the following constraint is ensured [13]

$$
\mathrm{Pe} \ll 4 \frac{d_{1}}{r_{v}},
$$

where $d_{1}$ is the distance between the transmitter and the closest receiver.

\section{B. Channel Model}

The probability for a molecule to be at a certain $d$ distance from the emission point at time $t$ is written as [13]

$$
p(d, t)=\frac{1}{\sqrt{4 \pi D_{e} t}} e^{-\frac{\left(d-v_{m} t\right)^{2}}{4 D_{e} t}},
$$

given that

$$
v_{m}=\frac{v_{\max }+v_{\min }}{2},
$$

where $v_{\max }$ is the maximum flow velocity (i.e., flow velocity in the center of the vessel) and $v_{\min }$ is the minimum flow velocity (i.e., $v_{\min }=0$ ), and

$$
D_{e}=D\left(1+\frac{1}{48} \mathrm{Pe}^{2}\right),
$$

where $D_{e}$ represents the effective diffusion coefficient. Note that validity of $D_{e}$ depends on the accuracy of (6).

By taking the integral of (7) between $d_{R, i}$ and $d_{R, i}+w$, we obtain the probability for a molecule to stand between $d_{R, i}$ and $d_{R, i}+w$ (i.e., to be sensed by $\Omega_{i}$, where $\Omega_{i}$ represents the $i$ th receiver) at a certain $t$ time as

$$
\mathbf{P}_{\mathrm{d}}\left(\Omega_{i}, t\right)=\operatorname{erf}\left(\frac{d_{R, i}+w-t v_{m}}{2 \sqrt{D_{e} t}}\right)-\operatorname{erf}\left(\frac{d_{R, i}-t v_{m}}{2 \sqrt{D_{e} t}}\right),
$$

where $d_{R, i}$ and $d_{R, i}+w$ are the starting and ending location of the $\Omega_{i}$ in the $x$ axis, respectively.

\section{LOCALIZATION}

We derive the analytical formulations for the localization of the transmitting cell both for known and unknown emission start times. Note that the derived formulations are independent from the location of the emission point in the $y$ and the $z$ axes.

\section{A. Scenario With Known Emission Start Time}

If the receiver knows the emission start time of the molecules, it can easily find $t_{p, 1}$, which is the mean peak time that the receiver observes the maximum number of molecules. 
Clearly, $t_{p, 1}$ can also be derived by taking the derivative of (10) w.r.t. time and equating it to zero as

$$
\begin{aligned}
& \left(d_{1}+v_{m} t_{p, 1}\right) \exp \left(\frac{-\left(d_{1}-v_{m} t_{p, 1}\right)^{2}}{4 D_{e} t_{p, 1}}\right) \\
& -\left(d_{1}+w+v_{m} t_{p, 1}\right) \exp \left(\frac{-\left(d_{1}+w-v_{m} t_{p, 1}\right)^{2}}{4 D_{e} t_{p, 1}}\right)=0 .
\end{aligned}
$$

Taking the natural logarithm of (11) yields,

$$
\frac{\left(d_{1}+w-v_{m} t_{p, 1}\right)^{2}-\left(d_{1}-v_{m} t_{p, 1}\right)^{2}}{4 D_{e} t_{p, 1}}=\log \left(1+\frac{w}{d_{1}+v_{m} t_{p, 1}}\right) \text {. }
$$

By approximating $\log (1+a) \simeq a$ for small values of $a$ with the assumption of $w \ll d_{1}+v_{m} t_{p, 1}$, we can rewrite (12) as

$$
2 d_{1}^{2}+w d_{1}+w v_{m} t_{p, 1}-2\left(v_{m} t_{p, 1}\right)^{2}-4 D_{e} t_{p, 1}=0,
$$

which can be solved as

$$
d_{1} \approx \frac{-w+\sqrt{w^{2}-8\left(w v_{m} t_{p, 1}-2\left(v_{m} t_{p, 1}\right)^{2}-4 D_{e} t_{p, 1}\right)}}{4} .
$$

Note that the negative root of (13) is not taken into account, since $w$ cannot be negative.

\section{B. Scenario With Unknown Emission Start Time}

It is possible that the receiver may not know the emission time and consequently cannot know the peak time $t_{p, 1}$ accurately (i.e., there is an offset time in the measured $t_{p, 1}$ ). This means that there are two unknowns as $t_{p, 1}$ and $d_{1}$ in (14). In order to solve this problem, we introduce a second receiver with width $w$, whose distance to the transmitter is $d_{2}$ and the mean peak time that second receiver observes the maximum number of molecules is $t_{p, 2}$. Note that we assume both receivers have the same width for the sake of simplicity; however, the proposed method also works in the case where the widths of receivers are not equal. Using these parameters, one can adapt (14) for the second receiver as

$$
d_{2} \approx \frac{-w+\sqrt{w^{2}-8\left(w v_{m} t_{p, 2}-2\left(v_{m} t_{p, 2}\right)^{2}-4 D_{e} t_{p, 2}\right)}}{4} .
$$

Note that both receivers do not know $t_{p, 1}, t_{p, 2}, d_{1}$ and $d_{2}$. However, they do know $\triangle t_{2,1}=\left(t_{p, 2}-t_{p, 1}\right)$ and $\triangle d_{2,1}=$ $\left(d_{2}-d_{1}\right)$. This knowledge implies that $t_{p, 2}=t_{p, 1}+\triangle t_{2,1}$ and $d_{2}=d_{1}+\triangle d_{2,1}$ in (15). Therefore, considering (14) and (15), $t_{p, 1}$ can be solved as

$$
t_{p, 1}=\frac{2 \triangle d_{2,1} \sqrt{H}-Y}{4\left(\triangle t_{2,1}^{2} v_{m}^{4}-\triangle d_{2,1}^{2} v_{m}^{2}\right)},
$$

where

$$
\begin{aligned}
H=\left(-4 D_{e}^{2}+2 D_{e} v_{m} w\right)\left(\triangle t_{2,1}^{2} v_{m}^{2}-\triangle d_{2,1}^{2}\right) \\
+\triangle t_{2,1}^{4} v_{m}^{6}-2 \triangle t_{2,1}^{2} \triangle d_{2,1}^{2} v_{m}^{4}+\triangle d_{2,1}^{4} v_{m}
\end{aligned}
$$

and

$$
\begin{aligned}
Y= & 4 D_{e}\left(\triangle t_{2,1}^{2} v_{m}^{2}+\triangle d_{2,1}^{2}\right)-2 \triangle t_{2,1}^{3} v_{m}^{4} \\
& +\triangle t_{2,1}^{2} v_{m}^{3} w+2 \triangle t_{2,1} \triangle d_{2,1}^{2} v_{m}^{2}-\triangle d_{2,1}^{2} v_{m} w .
\end{aligned}
$$

Once $t_{p, 1}$ is obtained, $d_{1}$ can be easily obtained by using (14).

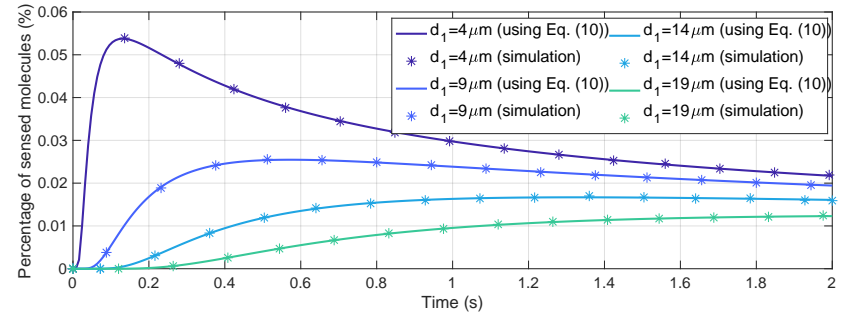

(a) $D=79.4 \mu \mathrm{m}^{2} / \mathrm{s}, r_{v}=5 \mu \mathrm{m}, v_{m}=0, w=1 \mu \mathrm{m}$

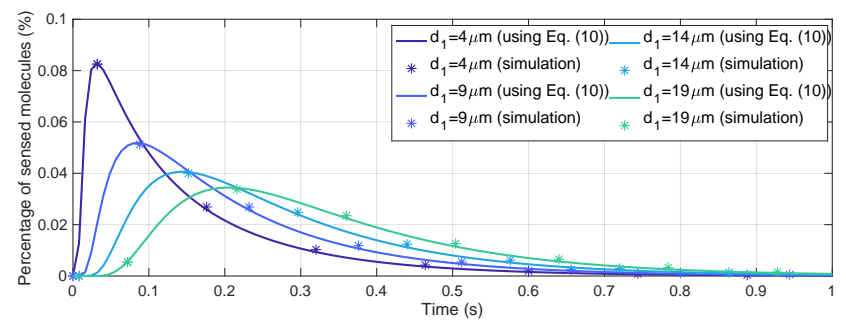

(b) $D=300 \mu \mathrm{m}^{2} / \mathrm{s}, r_{v}=2.5 \mu \mathrm{m}, v_{m}=80 \mu \mathrm{m} \mathrm{s}^{-1}, w=1 \mu \mathrm{m}$

Fig. 2. Time vs. percentage of sensed molecules.

\section{NuMERIC RESUlTS}

We have obtained the results using the analytical derivations (10), (14), (16), and a particle-based simulator, which keeps track of the MMs' movement in every simulation time step by evaluating the displacement of each MM at every time step as given in (1), (2), (3), and (4). By utilizing the outputs, we analyze the aforementioned channel and the two localization models under different conditions. Unless specified, number of emitted molecules $\left(n_{1}\right)$ is chosen as $10^{7}$, and simulation estimations are found by taking the average of 1000 simulation replications. The environment parameters are selected as in [1], [11], and $\Delta t$ is chosen as $0.1 \mathrm{~ms}$.

\section{A. Channel Model Analysis}

First of all, we have tested the validity of the proposed analytical derivation for the channel model in (10) by analyzing the percentage of the sensed molecules. The results, as given in Fig. 2, show that the analytical results match with the simulation results.

\section{B. Localization Analysis for Known Peak-time}

Fig. 3 shows the absolute errors in estimating the distance between the transmitter and the receiver using (14). Results show that absolute error decreases with increasing $d_{1}$ values, which better satisfy (6). Furthermore, in the conditions where (6) is not strongly satisfied (i.e., for small $d_{1}$ values), distances are estimated with relatively higher absolute errors. Note that the reason for estimating the distance erroneously, even when (6) is satisfied, is the $\log (1+a) \simeq a$ approximation. Also from upper lines to lower lines, $D_{e}$ values are increasing due to the increase in Pe, which in turn reduces the error. Note that $\mathrm{Pe}$ is increasing with the first power of $v_{m}$, whereas $D_{e}$ is increasing with the second power of $v_{m}$.

Fig. 4 shows the distance estimation results where the transmitter emits few molecules for which the peak times may be observed erroneously. Here, we compare the analytical results with the simulation estimates by varying $d_{1}$ and $n_{1}$, and using 


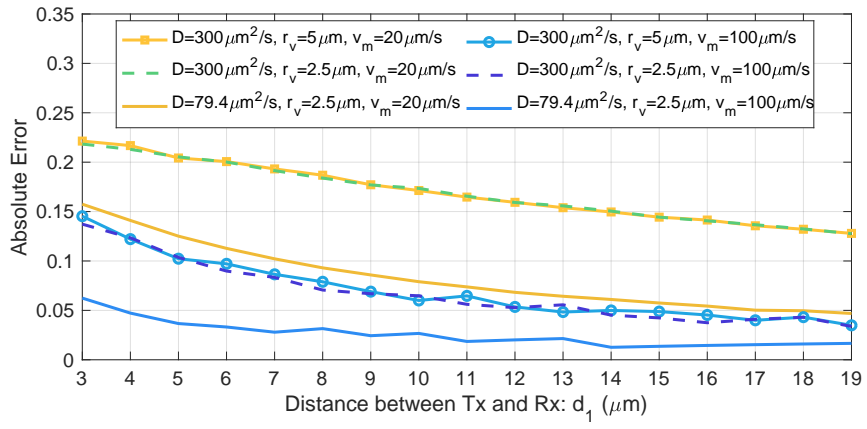

Fig. 3. Distance vs. absolute error for distance estimation considering emission start time is known (these results are obtained by using the analytical derivation in (14)) $(w=1 \mu \mathrm{m})$.

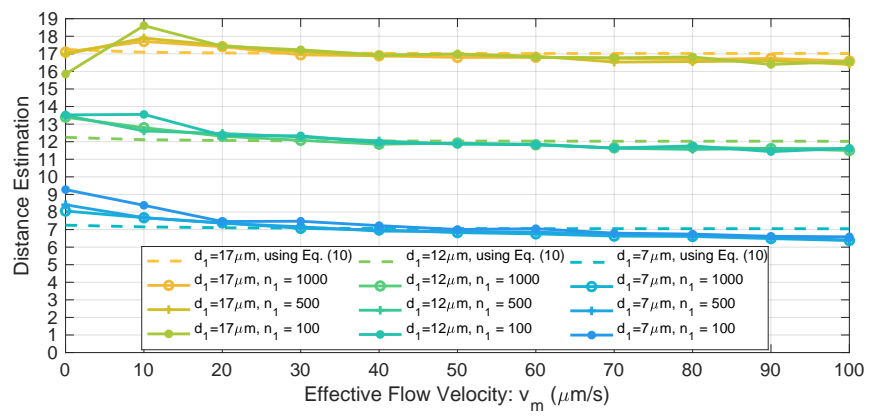

Fig. 4. Flow vs. distance estimation for two cases: (i) using $t_{p, 1}$, which is the analytical peak time of the observed molecules (ii) using $t_{p, 1}^{n_{1}}$, which is the peak time obtained by particle-based simulations assuming that $n_{1}$ molecules are emitted $\left(D=79.4 \mu \mathrm{m}^{2} / \mathrm{s}, r_{v}=5 \mu \mathrm{m}, w=1 \mu \mathrm{m}\right)$.

the measured peak time $t_{p, 1}^{\mathrm{n}_{1}}$ rather than $t_{p, 1}$. In the figure, the dashed and the solid lines represent the distance estimation results via analytical (10) and simulation methods. As can be seen in the figure, analytical estimations are pretty close to the actual distances. Distance estimations using simulations diverge slightly for small $v_{m}$ because the total amount of sensed data is not large enough to be statistically reliable to measure exact peak time. Moreover, distance estimations using smaller $n_{1}$ values diverge more than that for larger $n_{1}$ values. However, they converge back with increasing $v_{m}$ due to the increasing probability of sensing of the MMs. Also, distance estimations start to diverge after some certain $v_{m}$ values due to (6) no longer being satisfied.

\section{Localization Analysis for Unknown Peak-time}

When the emission start time is unknown, the peak time can be found using (16). After finding the peak time, the distance can be estimated using (14). In order to analyze the accuracy of these derived formulations, we select different pairs of receivers and compare the estimations. As can be seen from Fig. 5, estimations using the channel model in (10) diverge with the increasing $v_{m}$ values because the condition in (6) cannot be ensured for very large $v_{m}$ values.

\section{CONCLUSiOn And Future Work}

In this letter, we devise the analytical formulations to model a VLE and to localize the transmitter cell for different application scenarios in diffusion dominated movement. To that end, we first propose an analytical formulation for

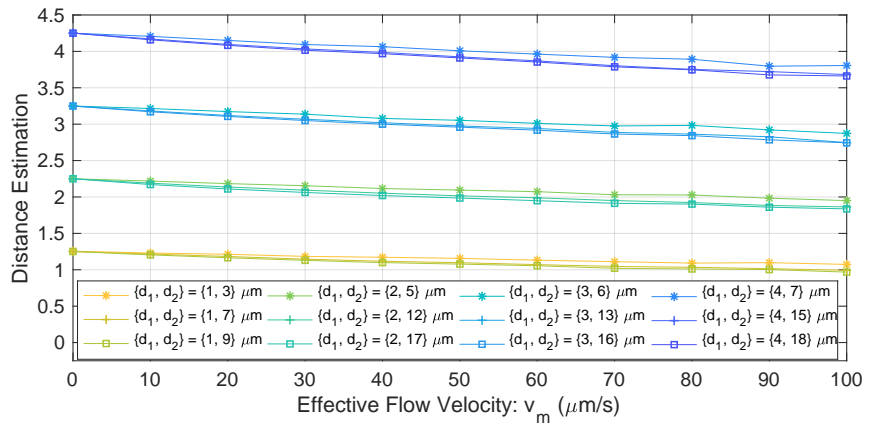

Fig. 5. Flow vs. distance estimation for receivers with different $d_{1}$ and $d_{2}$ values $\left(D=300 \mu \mathrm{m}^{2} / \mathrm{s}, r_{v}=5 \mu \mathrm{m}, w=1 \mu \mathrm{m}\right)$.

the channel model considering a point transmitter, observing receivers that have sensing capabilities through the inside of the vessel, and Poiseuille flow. Using this model, we have derived a formulation that calculates the distance between the transmitter and the receiver using a single receiver when the emission start time is known. We have also derived an analytical formulation for the unknown emission start time case by using two receivers. Finally, the devised analytical framework is validated using simulations.

As future work, we plan to estimate the distance between the transmitter and the receiver for also flow dominated movement, and consider more realistic channel including Casson effect, presence of the red blood cells, and cell free layer.

\section{REFERENCES}

[1] N. Farsad, H. B. Yilmaz, A. Eckford, C.-B. Chae, and W. Guo, "A comprehensive survey of recent advancements in molecular communication," IEEE Commun. Surveys Tuts., vol. 18, no. 3, pp. 1887-1919, 2016.

[2] T. Nakano, A. W. Eckford, and T. Haraguchi, Molecular Communication, 1st ed. Cambridge University Press, 2013.

[3] W. Guo, T. Asyhari, N. Farsad, H. B. Yilmaz, B. Li, A. Eckford, and C.-B. Chae, "Molecular communications: channel model and physical layer techniques," IEEE Wireless Communications, vol. 23, no. 4, pp. 120-127, August 2016.

[4] A. Noel, K. C. Cheung, and R. Schober, "Diffusive molecular communication with disruptive flows," in Proc. IEEE International Conference on Communications (ICC), June 2014.

[5] A. O. Bicen and I. F. Akyildiz, "System-theoretic analysis and leastsquares design of microfluidic channels for flow-induced molecular communication," IEEE Transactions on Signal Processing, vol. 61, no. 20, pp. 5000-5013, July 2013.

[6] J.-T. Huang, H.-Y. Lai, Y.-C. Lee, C.-H. Lee, and P.-C. Yeh, "Distance estimation in concentration-based molecular communications," in Global Communications Conference (GLOBECOM), 2013 IEEE. IEEE, 2013, pp. 2587-2591.

[7] X. Wang, M. D. Higgins, and M. S. Leeson, "Distance estimation schemes for diffusion based molecular communication systems," IEEE Communications Letters, vol. 19, no. 3, pp. 399-402, 2015.

[8] A. Noel, K. C. Cheung, and R. Schober, "Joint channel parameter estimation via diffusive molecular communication," IEEE Transactions on Molecular, Biological and Multi-Scale Communications, vol. 1, no. 1, pp. 4-17, 2015.

[9] L. Lin, C. Yang, S. Ma, and M. Ma, "Parameter estimation of inverse gaussian channel for diffusion-based molecular communication," in Wireless Communications and Networking Conference (WCNC), 2016 IEEE. IEEE, 2016, pp. 1-6.

[10] R. E. Klabunde, Cardiovascular Physiology Concepts, 2nd ed. Lippincott Williams \& Wilkins, 2012.

[11] D. E. Mohrman and L. J. Heller, Cardiovascular Physiology, 7th ed. McGraw-Hill Medical, 2010.

[12] H. Bruus, Theoretical Microfluidics, 1st ed. Oxford University Press, 2007.

[13] R. F. Probstein, Physicochemical Hydrodynamics: An Introduction, 2nd ed. Wiley-Interscience, 2003. 